\title{
A Nonlocal Strain Gradient Shell Model for Free Vibration Analysis of Functionally Graded Shear Deformable Nanotubes
}

\author{
Fahimeh Mehralian ${ }^{a}$ and Yaghoub Tadi Beni ${ }^{b^{*}}$ \\ ${ }^{a}$ Mechanical Engineering Depratment, Shahrekord University, Shahrekord, Iran \\ ${ }^{b}$ Faculty of Engineering, Shahrekord University, Shahrekord, Iran \\ E-mail address: tadi@eng.sku.ac.ir
}

Received date: April 2017

Accepted Date: May 2017

\begin{abstract}
In the current study, the size dependent free vibration of shear deformable functionally graded (FG) nanotubes is investigated. The nanotube is modeled as cylindrical shell which contains small scale effects by using the nonlocal strain gradient theory. Material properties of the FG nanotube are assumed to be variable along thickness direction according to power law distribution. The Hamilton's principle is implemented to derive the governing equations and boundary conditions. The numerical results are presented for simply supported FG nanotube and the influence of different parameters, such as nonlocal parameter, length scale parameter, length, thickness and power law index on frequency of FG nanotube are extensively studied. The results reveal that the frequency is significantly size dependent.
\end{abstract}

Keywords: Nonlocal strain gradient theory, Nanotube, Vibration, Size-dependent, first order shear deformation theory.

\section{Introduction}

Offering unique benefits compared to conventional materials, functionally graded materials have been found tremendous amount of interest among researchers. The material properties of FG materials are changed smoothly in one or more directions to overcome stress concentration, as a common problem in usual composite materials [1]. Since they include two different components, FG materials are able to utilize the desirable properties of each constituent and as a result they can be designed for specific functions and applications. The static and dynamic behavior of FG beams, plats and shells are studied by many researchers. For example, Tadi et al. studied the free vibration of FG nanoshells and the effects of different parameters on frequency was shown as well [2]. The bending, buckling and vibration behaviors of axially FG nanobeams were investigated by $\mathrm{Li}$ et al and the critical buckling force and natural frequency were shown size dependent [3]. Ebrahimi et al. examined the wave propagation of FG nanoplate under nonlinear thermal loading and the influence of different parameters such as gradient index, temperature distribution and length scale parameter on the wave dispersion was presented [4]. The buckling of cylindrical and conical panels and shells of laminated composite, FGM and carbon nanotube reinforced functionally graded cases were examined by Civalek and the effects of material and geometrical parameters on buckling response were shown [5]. Akgöz et al. studied the longitudinal free vibration of axially FG microbars for different boundary conditions and the effect of material and geometrical parameters on natural frequency was shown [6]. 
In recent years, the increasing growth of nanotechnology leads to inspiring innovations in electrical, magnetic, and optical devices at the nanoscale and nanotubes are surely the most exciting nanostructure playing an important role in nanotechnology today [7]. The research on nanotubes has illustrated their prominent mechanical and electronic properties which are expected to result in revolutionary new devices. The more accurate realization of nanotubes behavior, however has so far been limited because of their dimensions, which are often equal or smaller than the characteristics length scales [8]. Modified continuum theories, which are developed as analytical methods producing more accurate results as such being comparable to those of atomistic models, are utilized in many studies. For example, Mehralian et al. studied the buckling of FG piezoelectric nanoshell under pressure based on the new modified couple stress theory and the critical buckling pressure was shown significantly size dependent by increase in thickness and decrease in length [9]. Size-dependent first order shear deformable shell model on the basis of modified strain gradient theory was utilized by Gholami et al. to study the axial buckling of functionally graded cylindrical shell [10]. The effect of material property gradient index was illustrated significant on the buckling load. Mehralian et al. studied the free vibration of FG truncated conical shell in thermal environment based on the modified couple stress theory and natural frequency was shown significantly size dependent particularly by decreasing apex angle and increasing gradient index [11]. The size dependent buckling behavior of silicon carbide nanotubes were investigated by Mercan et al. on the basis of Eringen's nonlocal elasticity and surface elasticity and the influence of geometrical parameters on critical buckling load was indicated [12]. Akgöz et al. studied the buckling of single walled carbon nanotubes using modified couple stress theory and strain gradient theory [13].

Nonlocal strain gradient theory, as higher order continuum theory, which is able to predict the stiffens-hardening effects besides stiffness-softening ones, is introduced by Lim et al. [14]. In this theory, the stress field accounts nonlocal stress field besides strain gradients stress filed and two material length scale parameters beside two Lame constants are introduced [14]. There are many studies in which the static and dynamic behaviors of nanobeams and nanoplates are investigated based on this theory. For example, Ebrahimi et al. examined the buckling of curved FG nanobeam based on the nonlocal strain gradient theory for simply supported and clamped boundary conditions and the effect of different parameters such as length scale parameters, power law exponent and boundary conditions were indicated [15]. The wave propagation in a viscoelastic SWCNT are studied based on the nonlocal strain gradient theory using Timoshenko beam model by Tang et al. and the effects of tube size on the wave dispersion was shown [16].

Motivated by the mentioned discussion, this paper examines the vibration of FG nanotube based on the nonlocal strain gradient theory using the first order shear deformation shell model. The governing equations and boundary conditions are derived using Hamilton's principle. The free vibration of simply supported cylindrical shell, as a case study, is investigated. The effects of different parameters such as material length scale parameters, thickness ratio and length ratio are illustrated on the frequency.

\section{Theoretical development}

Consider a FG nanotube modeled as cylindrical shell in Fig. 1, in which geometrical parameters of length, $L$, radius, $R$ and thickness $h$ are also indicated. FGM is usually made by the combination of two components (e.g. ceramics and metal) and the material properties of FG cylindrical shell varies continuously and consistently from the material properties of ceramics on the inner surface of the cylindrical shell to the properties of the metal on the outer surface as a function of constituent's volume fraction. Variation in volume fraction of metal 
and ceramic according to power law distribution along cylindrical shell thickness is expressed in the following equations:

$$
\begin{aligned}
V_{m} & =\left(\frac{\hat{z}}{h}\right)^{\beta} \\
V_{c} & =1-V_{m}
\end{aligned}
$$

In the above equation, $\beta$ stands for power index which varies in the $0 \leq \beta \leq \infty$ interval, and as illustrated by Fig. 1, $\hat{z}$ stands for the arbitrary surface distance from the inner ones of the cylindrical shell. Therefore, the material properties of this cylindrical shell are expressed as:

$$
\begin{aligned}
& E(\hat{z})=\left(E_{m}-E_{c}\right)\left(\frac{\hat{z}}{h}\right)^{\beta}+E_{c} \\
& \rho(\hat{z})=\left(\rho_{m}-\rho_{c}\right)\left(\frac{\hat{z}}{h}\right)^{\beta}+\rho_{c} \\
& v(\hat{z})=\left(v_{m}-v_{c}\right)\left(\frac{\hat{z}}{h}\right)^{\beta}+v_{c}
\end{aligned}
$$

where $E_{c}, \rho_{\mathrm{c}}$ and $v_{\mathrm{c}}$ are obtained in $\hat{z}=0$, and $E_{m}, \rho_{\mathrm{m}}$ and $v_{\mathrm{m}}$ are obtained in $\hat{z}=h$, which respectively represent Young's modulus, density and Poisson's ratio of ceramics and metal.

As displayed by Fig. 1, the displacement field of cylindrical shell based on first order shear deformation theory along the three directions of $x, \theta$ and $z$ is expressed as:

$$
\begin{gathered}
U(x, \theta, z, t)=u(x, \theta, t)+z \psi_{x}(x, \theta, t) \\
V(x, \theta, z, t)=v(x, \theta, t)+z \psi_{\theta}(x, \theta, t) \\
W(x, \theta, z, t)=w(x, \theta, t)
\end{gathered}
$$

In the above equation, $u(x, \theta, t), v(x, \theta, t)$ and $w(x, \theta, t)$ are considered as neutral axis displacement, and $\psi_{x}(x, \theta, t)$ and $\psi_{\theta}(x, \theta, t)$ as rotation of a transverse normal about the circumferential and axial directions. Besides, the position of the neutral axis is expressed as follows [2]:

$$
\hat{z}_{c}=\frac{\int_{A} \frac{E(\hat{z})}{1-v^{2}(\hat{z})} \hat{z} d A}{\int_{A} \frac{E(\hat{z})}{1-v^{2}(\hat{z})} d A}
$$

To extract the governing equations of FG nanotubes, Hamilton's principle is utilized as below:

$$
\int_{\Delta t}\left(\delta U_{s}-\delta T+\delta W_{e}\right) d t=0
$$


where $\delta T$ represents kinetic energy variation, $\delta U_{s}$ stands for strain energy variation, and $\delta W_{e}$ is variation in the work of external loads acting on the cylindrical shell, which is neglected in this study .

The kinetic energy is obtained from time derivation on the displacement variables, as follows:

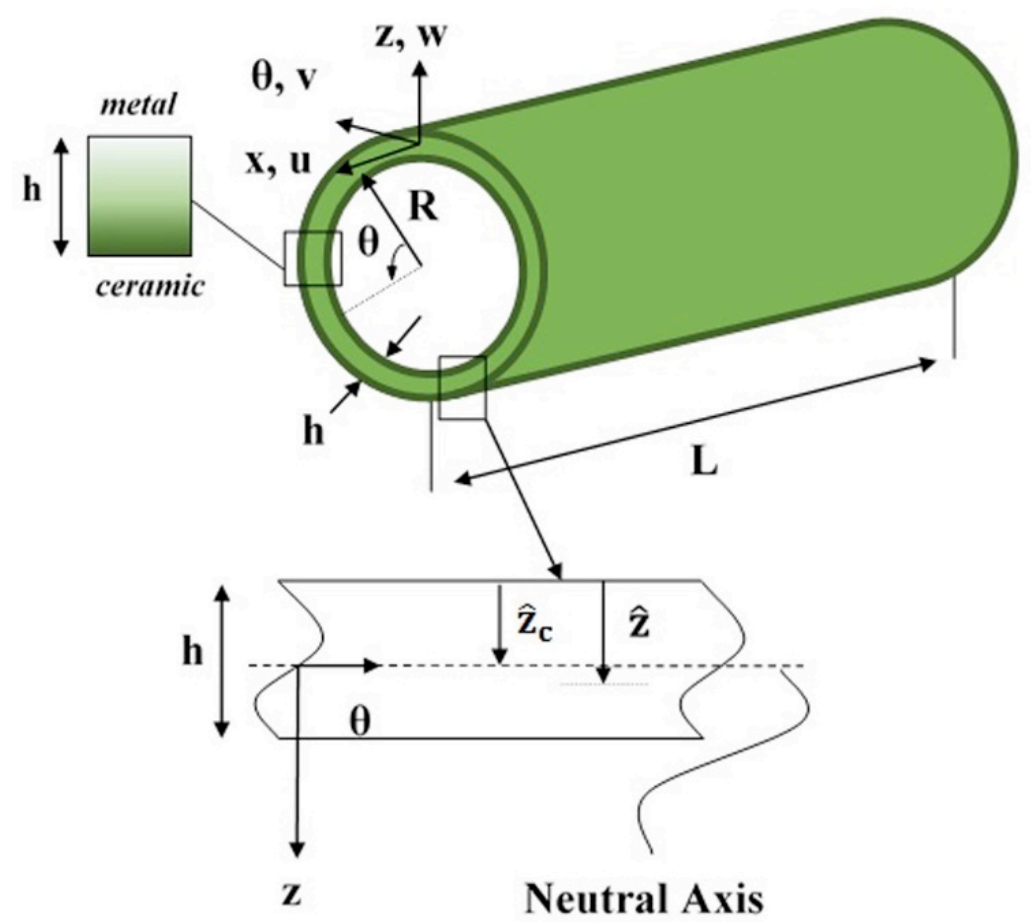

Fig. 1. Coordinate system and geometry of the FG nanotube.

$$
T=\frac{\rho(\hat{z})}{2} \iiint_{V}\left[U^{2}+V^{2}+W^{2}\right] d V
$$

and the variation of kinetic energy is obtained as:

$$
\delta T=\frac{1}{2} \int_{V} \rho(\hat{z})\left[\left(\frac{\partial \delta u}{\partial t}+z \frac{\partial \delta \psi_{x}}{\partial t}\right)^{2}+\left(\frac{\partial \delta v}{\partial t}+z \frac{\partial \delta \delta \psi_{\theta}}{\partial t}\right)^{2}+\left(\frac{\partial \delta w}{\partial t}\right)^{2}\right] R d x d \theta d z
$$

Based on the nonlocal strain gradient theory proposed by Lim et al. the strain energy is given by [9]:

$$
U_{s}=\frac{1}{2} \iiint_{V}\left(\sigma_{i j} \varepsilon_{i j}+\sigma_{i j}^{(1)} \nabla \varepsilon_{i j}\right) d V
$$

where

$$
\begin{gathered}
\varepsilon_{i j}=\frac{1}{2}\left(u_{i, j}+u_{j, i}\right) \\
\sigma_{i j}=C_{i j k l} \varepsilon_{k l}
\end{gathered}
$$


which, $\varepsilon_{i j}, \sigma_{i j}$ are the components of strain and stress tensor respectively and $C_{i j k l}$ represents the elasticity tensor for cylindrical shell. Also, the non-zero components of strain field are obtained by substituting Eq. (3) into (9) and using the assumption $(1+z / R) \approx 1$.

$$
\begin{gathered}
\varepsilon_{x x}=\frac{\partial u}{\partial x}+z \frac{\partial \psi_{x}}{\partial x} \\
\varepsilon_{\theta \theta}=\frac{1}{R}\left(\frac{\partial v}{\partial \theta}+w+z \frac{\partial \psi_{\theta}}{\partial \theta}\right) \\
\varepsilon_{x \theta}=\frac{1}{2}\left(\frac{1}{R} \frac{\partial u}{\partial \theta}+\frac{\partial v}{\partial x}+\frac{z}{R} \frac{\partial \psi_{x}}{\partial \theta}+z \frac{\partial \psi_{\theta}}{\partial x}\right) \\
\varepsilon_{z x}=\frac{1}{2}\left(\psi_{x}+\frac{\partial w}{\partial x}\right) \\
\varepsilon_{z \theta}=\frac{1}{2}\left(\psi_{\theta}+\frac{1}{R} \frac{\partial w}{\partial \theta}-\frac{v}{R}\right)
\end{gathered}
$$

Given the assumption of plane stress in the shear deformation shell theory, the stress tensor can be defined as:

$$
\begin{gathered}
\left\{\begin{array}{c}
\sigma_{x x} \\
\sigma_{\theta \theta} \\
\sigma_{x \theta}
\end{array}\right\}=\left[\begin{array}{ccc}
C_{11} & C_{12} & 0 \\
C_{12} & C_{22} & 0 \\
0 & 0 & C_{66}
\end{array}\right]\left\{\begin{array}{c}
\varepsilon_{x x} \\
\varepsilon_{\theta \theta} \\
2 \varepsilon_{x \theta}
\end{array}\right\} \\
\left\{\begin{array}{l}
\sigma_{x z} \\
\sigma_{\theta z}
\end{array}\right\}=\left[\begin{array}{cc}
C_{44} & 0 \\
0 & C_{55}
\end{array}\right]\left\{\begin{array}{l}
2 \varepsilon_{x z} \\
2 \varepsilon_{\theta z}
\end{array}\right\}
\end{gathered}
$$

In Eq. (12), elastic constants are defined as:

$$
C_{11}=C_{22}=\frac{E(\hat{z})}{1-v(\hat{z})^{2}}, \quad C_{12}=\frac{E(\hat{z}) v(\hat{z})}{1-v(\hat{z})^{2}}, \quad C_{33}=C_{44}=C_{55}=\mu(\hat{z})
$$

In the above equation, $E(\hat{z})$ and $v(\hat{z})$ respectively represent Young's modulus and Poisson's ratio for FG cylindrical shell. Also, by substituting Eqs. (11) and (12) into Eq. (8), the variation of strain energy is obtained:

$$
\int_{d V} \delta U_{s} d V=\int_{d V}\left[t_{i j}\right] \delta \varepsilon_{i j} d V+\int_{d A}\left[\sigma_{i j}^{(1)}\right] \delta \varepsilon_{i j} \mid \begin{aligned}
& L \\
& 0
\end{aligned} d A
$$

where

$$
t_{i j}=\sigma_{i j}-\nabla \sigma_{i j}^{(1)}
$$

According to nonlocal strain gradient theory, its constitutive equation is as follows: 


$$
\left[1-\mu \nabla^{2}\right] t_{i j}=C_{i j k l} \varepsilon_{k l}-\eta C_{i j k l} \nabla^{2} \varepsilon_{k l}
$$

In the above equation, $\mu$ is equal to square of nonlocal scale parameter $\left(e_{0} a\right)$. Furthermore, $\eta$ is equal to square of material length scale parameter $(l)$.

Consequently, by substituting Eqs. $(7,14)$ into Eq. (5) and calculating multiple integral by parts, the governing equations of FG nanotube are extracted as:

$$
\begin{aligned}
& \delta U:\left(1-\eta \nabla^{2}\right)\left[A_{1} \frac{\partial^{2}}{\partial x^{2}} u(x, \theta, t)+A_{2} \frac{\partial^{2}}{\partial x^{2}} \psi_{x}(x, \theta, t)+A_{3} \frac{\partial^{2}}{\partial x \partial \theta} v(x, \theta, t)\right. \\
& \left.+A_{4} \frac{\partial}{\partial x} w(x, \theta, t)+A_{5} \frac{\partial^{2}}{\partial x \partial \theta} \psi_{\theta}(x, \theta, t)+A_{6} \frac{\partial^{2}}{\partial \theta^{2}} u(x, \theta, t)+A_{7} \frac{\partial^{2}}{\partial \theta^{2}} \psi_{x}(x, \theta, t)\right] \\
& -\left(1-\mu \nabla^{2}\right)\left[A_{8} \frac{\partial^{2}}{\partial t^{2}} u(x, \theta, t)+A_{9} \frac{\partial^{2}}{\partial t^{2}} \psi_{x}(x, \theta, t)\right]=0 \\
& \delta V:\left(1-\eta \nabla^{2}\right)\left[B_{1} \frac{\partial^{2}}{\partial x \partial \theta} u(x, \theta, t)+B_{2} \frac{\partial^{2}}{\partial x^{2}} v(x, \theta, t)+B_{3} \frac{\partial^{2}}{\partial x \partial \theta} \psi_{x}(x, \theta, t)\right. \\
& +B_{4} \frac{\partial^{2}}{\partial x^{2}} \psi_{\theta}(x, \theta, t)+B_{5} \frac{\partial^{2}}{\partial \theta^{2}} v(x, \theta, t)+B_{6} \frac{\partial}{\partial \theta} w(x, \theta, t) \\
& \left.+B_{7} \frac{\partial^{2}}{\partial \theta^{2}} \psi_{\theta}(x, \theta, t)+B_{8} \psi_{\theta}(x, \theta, t)+B_{9} v(x, \theta, t)\right] \\
& -\left(1-\mu \nabla^{2}\right)\left[B_{10} \frac{\partial^{2}}{\partial t^{2}} v(x, \theta, t)+B_{11} \frac{\partial^{2}}{\partial t^{2}} \psi_{\theta}(x, \theta, t)\right]=0 \\
& \delta W:\left(1-\eta \nabla^{2}\right)\left[C_{1} \frac{\partial}{\partial x} \psi_{x}(x, \theta, t)+C_{2} \frac{\partial^{2}}{\partial x^{2}} w(x, \theta, t)+C_{3} \frac{\partial}{\partial \theta} \psi_{\theta}(x, \theta, t)\right. \\
& \left.+C_{4} \frac{\partial^{2}}{\partial \theta^{2}} w(x, \theta, t)+C_{5} \frac{\partial}{\partial \theta} v(x, \theta, t)+C_{6} w(x, \theta, t)+C_{7} \frac{\partial}{\partial x} u(x, \theta, t)\right] \\
& -\left(1-\mu \nabla^{2}\right)\left[C_{8} \frac{\partial^{2}}{\partial t^{2}} w(x, \theta, t)\right]=0 \\
& \delta \psi_{x}:\left(1-\eta \nabla^{2}\right)\left[D_{1} \frac{\partial^{2}}{\partial x^{2}} u(x, \theta, t)+D_{2} \frac{\partial^{2}}{\partial x^{2}} \psi_{x}(x, \theta, t)+D_{3} \frac{\partial^{2}}{\partial x \partial \theta} v(x, \theta, t)\right. \\
& +D_{4} \frac{\partial}{\partial x} w(x, \theta, t)+D_{5} \frac{\partial^{2}}{\partial x \partial \theta} \psi_{\theta}(x, \theta, t)+D_{6} \frac{\partial^{2}}{\partial \theta^{2}} u(x, \theta, t)+D_{7} \frac{\partial^{2}}{\partial \theta^{2}} \psi_{x}(x, \theta, t) \\
& \left.+D_{8} \psi_{x}(x, \theta, t)\right]-\left(1-\mu \nabla^{2}\right)\left[D_{9} \frac{\partial^{2}}{\partial t^{2}} \psi_{x}(x, \theta, t)+D_{10} \frac{\partial^{2}}{\partial t^{2}} u(x, \theta, t)\right]=0
\end{aligned}
$$




$$
\begin{aligned}
& \delta \psi_{\theta}:\left[E_{1} \frac{\partial^{2}}{\partial x \partial \theta} u(x, \theta, t)+E_{2} \frac{\partial^{2}}{\partial x^{2}} v(x, \theta, t)+E_{3} \frac{\partial^{2}}{\partial x \partial \theta} \psi_{x}(x, \theta, t)+E_{4} \frac{\partial^{2}}{\partial x^{2}} \psi_{\theta}(x, \theta, t)\right. \\
& +E_{5} \frac{\partial^{2}}{\partial \theta^{2}} v(x, \theta, t)+E_{6} \frac{\partial}{\partial \theta} w(x, \theta, t)+E_{7} \frac{\partial^{2}}{\partial \theta^{2}} \psi_{\theta}(x, \theta, t)+E_{8} \psi_{\theta}(x, \theta, t) \\
& \left.+E_{9} v(x, \theta, t)\right]-\left(1-\mu \nabla^{2}\right)\left[E_{10} \frac{\partial^{2}}{\partial t^{2}} \psi_{\theta}(x, \theta, t)+E_{11} \frac{\partial^{2}}{\partial t^{2}} v(x, \theta, t)\right]=0
\end{aligned}
$$

where

$$
\begin{aligned}
& A_{1}=Y_{1}, A_{2}=Y_{2}, A_{3}=Y_{4} / R+Y_{7} / R, A_{4}=Y_{4} / R, A_{5}=Y_{5} / R+Y_{8} / R, \\
& A_{6}=Y_{7} / R^{2}, A_{7}=Y_{8} / R^{2}, A_{8}=Y_{10}, A_{9}=Y_{11} \\
& B_{1}=Y_{7} / R+Y_{4} / R, B_{2}=Y_{7}, B_{3}=Y_{8} / R+Y_{5} / R, B_{4}=Y_{8}, B_{5}=Y_{1} / R^{2}, \\
& B_{6}=Y_{1} / R^{2}+k Y_{7} / R^{2}, B_{7}=Y_{2} / R^{2}, B_{8}=k Y_{7} / R, B_{9}=-k Y_{7} / R^{2}, B_{10}=Y_{10}, B_{11}=Y_{11} \\
& C_{1}=k Y 7-Y 5 / R, C_{2}=k Y_{7}, C_{3}=k Y_{7} / R-Y_{2} / R^{2}, C_{4}=k Y_{7} / R^{2}, C_{5}=-k Y_{7} / R^{2}-Y_{1} / R^{2}, \\
& C_{6}=-Y_{1} / R^{2}, C_{7}=-Y_{4} / R, \mathrm{C}_{8}=Y_{10} \\
& D_{1}=Y_{2}, D_{2}=Y_{3}, D_{3}=Y_{5} / R+Y_{8} / R, D_{4}=Y_{5} / R-k Y_{7}, D_{5}=Y_{6} / R+Y_{9} / R, D_{6}=Y_{8} / R^{2}, \\
& D_{7}=Y_{9} / R^{2}, D_{8}=-k Y_{7}, D_{9}=Y_{12}, D_{10}=Y_{11} \\
& E_{1}=Y_{8} / R+Y_{5} / R, E_{2}=Y_{8}, E_{3}=Y_{9} / R+Y_{6} / R, E_{4}=Y_{9}, E_{5}=Y_{2} / R^{2}, E_{6}=Y_{2} / R^{2}-k Y_{7} / R, \\
& E_{7}=Y_{3} / R^{2}, E_{8}=-k Y_{7}, E_{9}=k Y_{7} / R, E_{10}=Y_{12}, E_{11}=Y_{11}
\end{aligned}
$$

and

$$
\begin{aligned}
& Y_{i}=\int_{-\hat{z}_{c}}^{h-\hat{z}_{c}} \frac{E(\hat{z})}{1-v^{2}(\hat{z})}\left(z^{i}\right) d z,(i=1,2,3), Y_{i}=\int_{-\hat{z}_{c}}^{h-\hat{z}_{c}} \frac{E(\hat{z}) v(\hat{z})}{1-v^{2}(\hat{z})}\left(z^{i}\right) d z,(i=4,5,6), \\
& Y_{i}=\int_{-\hat{z}_{c}}^{h-\hat{z}_{c}} \mu(\hat{z})\left(z^{i}\right) d z,(i=7,8,9), Y_{i}=\int_{-\hat{z}_{c}}^{h-\hat{z}_{c}} \rho(\hat{z})\left(z^{i}\right) d z,(i=10,11,12)
\end{aligned}
$$

The boundary conditions are given in Appendix A.

In order to solve the governing equations, the following approximate solutions, satisfied differential equations and boundary conditions, are utilized:

$$
\begin{aligned}
u(x, \theta, \mathrm{t}) & =\sum_{n} \sum_{m} U_{m n}(t) \cos \left(\frac{m \pi x}{L}\right) \cos (n \theta) \\
v(x, \theta, \mathrm{t}) & =\sum_{n} \sum_{m} V_{m n}(t) \sin \left(\frac{m \pi x}{L}\right) \sin (n \theta) \\
w(x, \theta, \mathrm{t}) & =\sum_{n} \sum_{m} W_{m n}(t) \sin \left(\frac{m \pi x}{L}\right) \cos (n \theta) \\
\psi_{x}(x, \theta, \mathrm{t}) & =\sum_{n} \sum_{m} \psi_{x m n}(t) \cos \left(\frac{m \pi x}{L}\right) \cos (n \theta)
\end{aligned}
$$




$$
\psi_{\theta}(x, \theta, \mathrm{t})=\sum_{n} \sum_{m} \psi_{\theta m n}(t) \sin \left(\frac{m \pi x}{L}\right) \sin (n \theta)
$$

where $m, n$ stand for axial and circumferential wave numbers.

Therefore, by substituting Eq. (22) into the equations of motion, the equations are written in the matrix form as follows:

$$
[k]\{d\}+[M]\{d\}=0
$$

where

$$
\{d\}=\left\{d_{0}\right\} e^{i \omega t}
$$

Now, by substituting Eq. (24) into (23), we have

$$
\left([k]-\omega^{2}[M]\right)\left\{d_{0}\right\}=0
$$

where $\omega$ stands for natural frequency, $\left\{d_{0}\right\}=\left\{U_{m n} V_{m n} W_{m n} \psi_{x m n} \psi_{\theta m n}\right\}^{T}$ is displacement amplitude vector. To obtain the non-trivial solution to Eq. (25), one must consider the determinant of coefficients equivalent to zero from which the shell frequency equation is derived and solved.

\section{Results}

For the sake of predicting the vibration behavior of nanotubes more accurately using nonlocal strain gradient theory, since the efficiency of the nonlocal strain gradient shell model is strongly dependent on the recognition of the proper values of small length scale parameters, $\mu$ $=\left(e_{0} a\right)^{2}$ and $\eta=l^{2}$ are also calibrated using MD results of a $(5,5)$ armchair CNT, due to lacking of the values of small length scale parameters of FG nanotubes. Also the values of $\mu$ and $\eta$ are considered to be $(3.3)^{2}$ to $(3.5)^{2} \mathrm{~nm}^{2}$ and $(0.1)^{2}$ to $(0.4)^{2} \mathrm{~nm}^{2}$, respectively, for different length ratios. The following material parameters are considered for FG nanotube [17]:

Table 1. Material properties of FG cylindrical shell.

\begin{tabular}{cccc}
\hline & $E(\mathrm{GPa})$ & $v$ & $\rho\left(\mathrm{kg} / \mathrm{m}^{3}\right)$ \\
\hline Aluminum & 70 & 0.3 & 2702 \\
Ceramics & 427 & 0.17 & 3100 \\
\hline
\end{tabular}

In the following, the vibration response of nanotubes under different material and geometrical parameters is indicated to illustrate the applications of nonlocal strain gradient theory.

In order to show the influences of small length scale parameters on frequency of nanotubes, Figs. 2 and 3 are presented. It is seen that increasing nonlocal parameter $(\mu)$ at a certain scale factor $(\eta)$ decreases frequency which reveals the softening effect of nonlocal parameter (see Fig. 2); while, increasing scale factor in the case of certain nonlocal parameter increases frequency and it means that the effective stiffness of nanotube becomes larger with increasing scale factor (see Fig. 3). These phenomena illustrate that by using nonlocal strain gradient theory, the nanotube exerts the softening and stiffening behavior by increasing the nonlocal 
parameter and scale factor, respectively. Besides, due to the higher elastic modulus of ceramics compared to aluminum, with the increase in the gradient index in the shell, where $\beta$ $=0$ is for the aluminum shell and $\beta=\infty$ for the ceramic shell, the frequency increases as well.

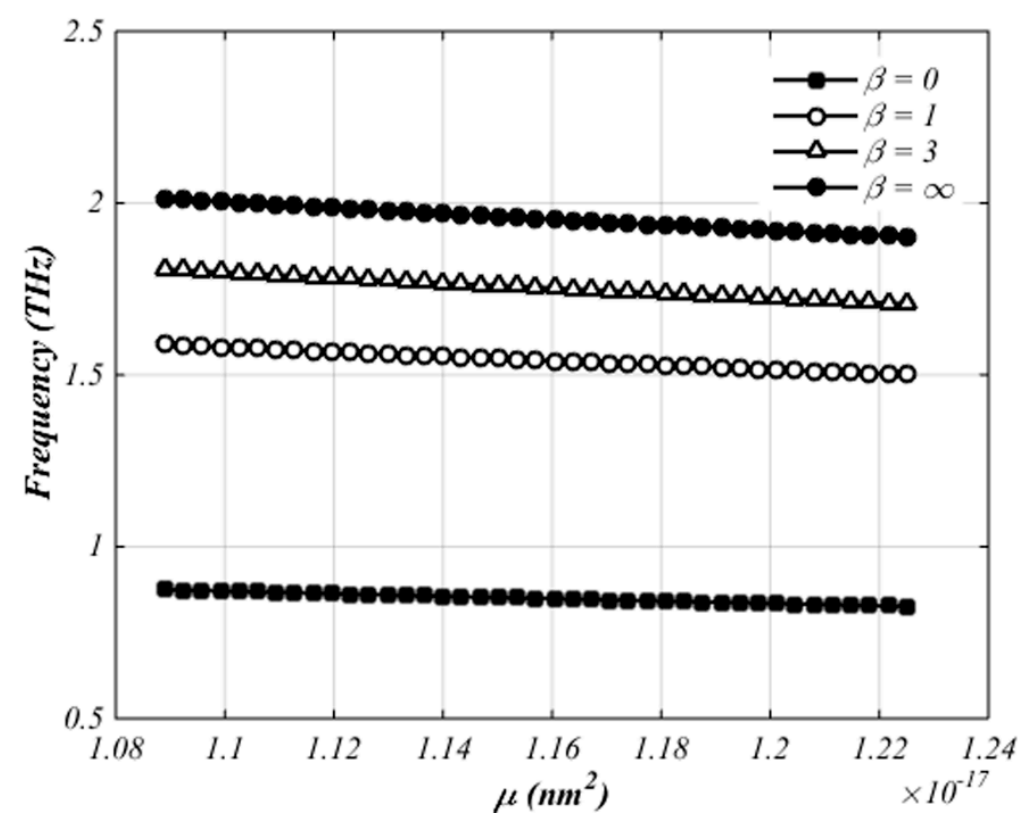

Fig. 2. Effect of nonlocal parameter on frequency for different power law index.

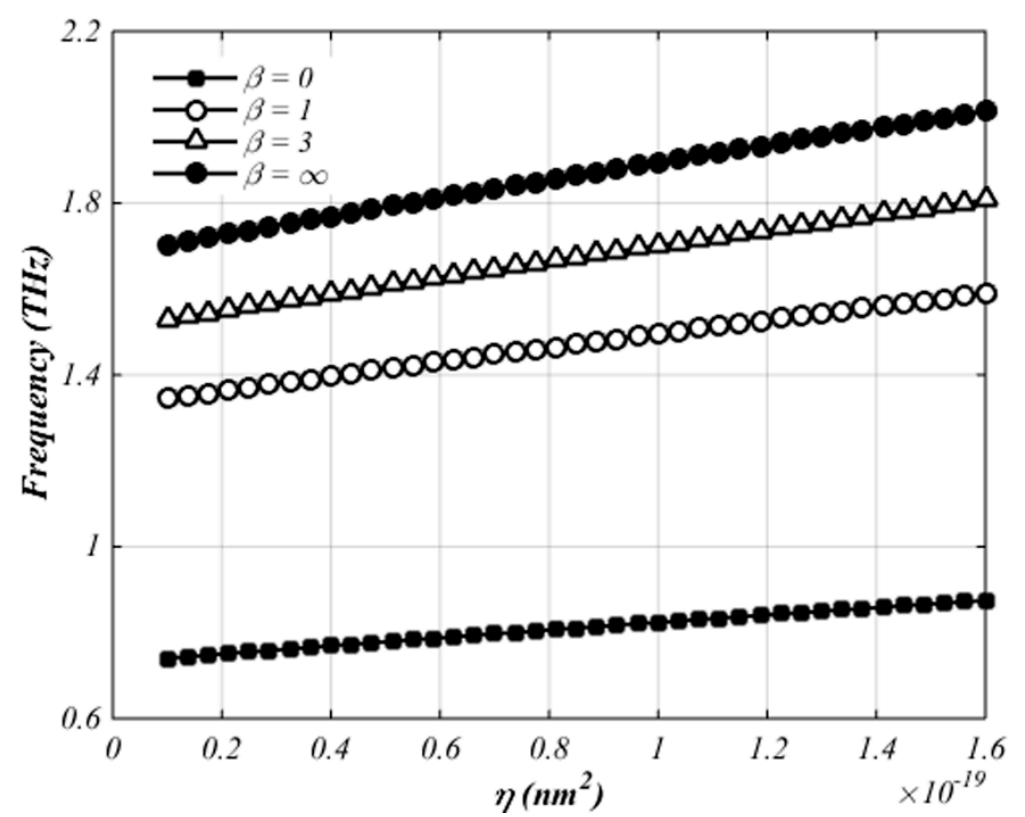

Fig. 3. Effect of scale factor on frequency for different power law index.

Fig. 4 is indicated the influences of thickness ratio on frequency of nanotubes. Regarding Fig. 4 , it is witnessed that the increase in thickness ratio contributes to the higher frequency for various values of power law index because of ascending the stiffness of nanotube; besides, the more increase in the frequency is occurred when the power law index goes up. Also, it is found that the higher frequency takes place at high power law index and thickness ratio. This is regarded as evidence that the power law index makes nanotube stiffer.

In order to see the effects of thickness ratio more clearly, Figs. 5 and 6 illustrate the effects of thickness ratio on frequency of nanotubes, particularly on different scale factors and nonlocal 
parameters. It is shown that, the high frequency appears at high scale factor and low nonlocal parameter. It is clear that the trends of the frequency variation versus thickness ratio for various scale factors and nonlocal parameters are similar to Fig. 4 and similar conclusion can be drawn. It should be noted that, the influence of the transverse shear deformation is significant when thick and short nanotubes are investigated and since the first order shear deformation theory is used in this study, there is no limitation on choosing the values of thickness parameter.

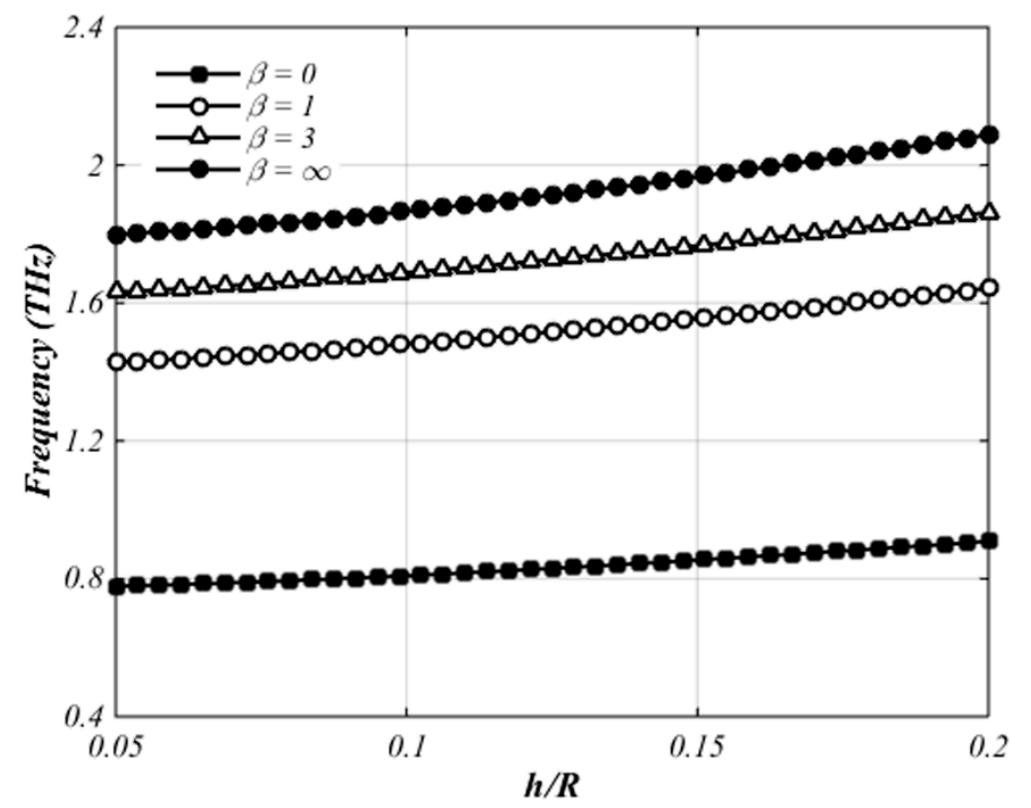

Fig. 4. Effect of thickness ratio on the frequency for different power law index $\left(\mu=(3.3 e-9)^{2}, \eta=\right.$ $\left.(0.4 \mathrm{e}-9)^{2}\right)$.

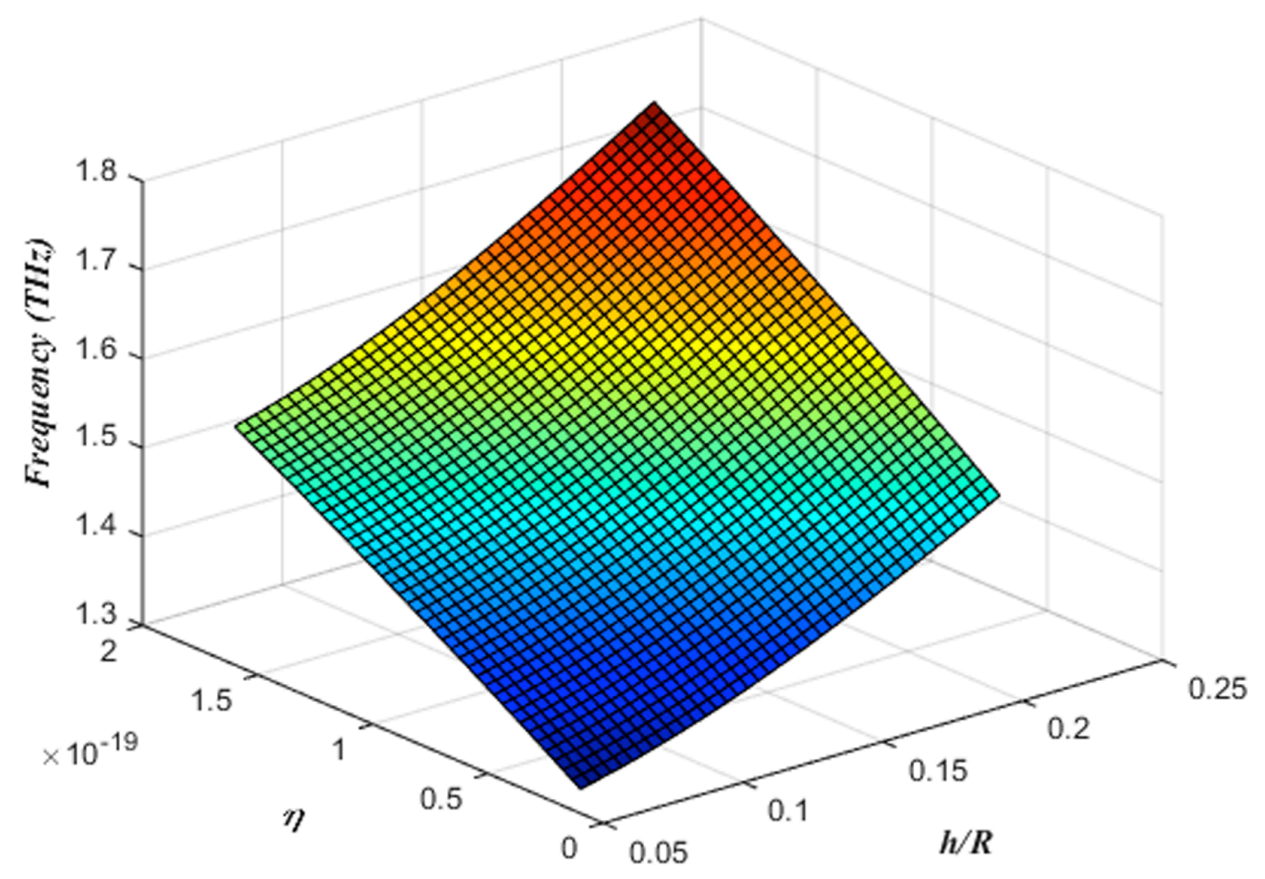

Fig. 5. Effect of thickness ratio on the frequency for different scale factors $\left(\beta=2, \mu=(3.3 e-9)^{2}\right)$. 


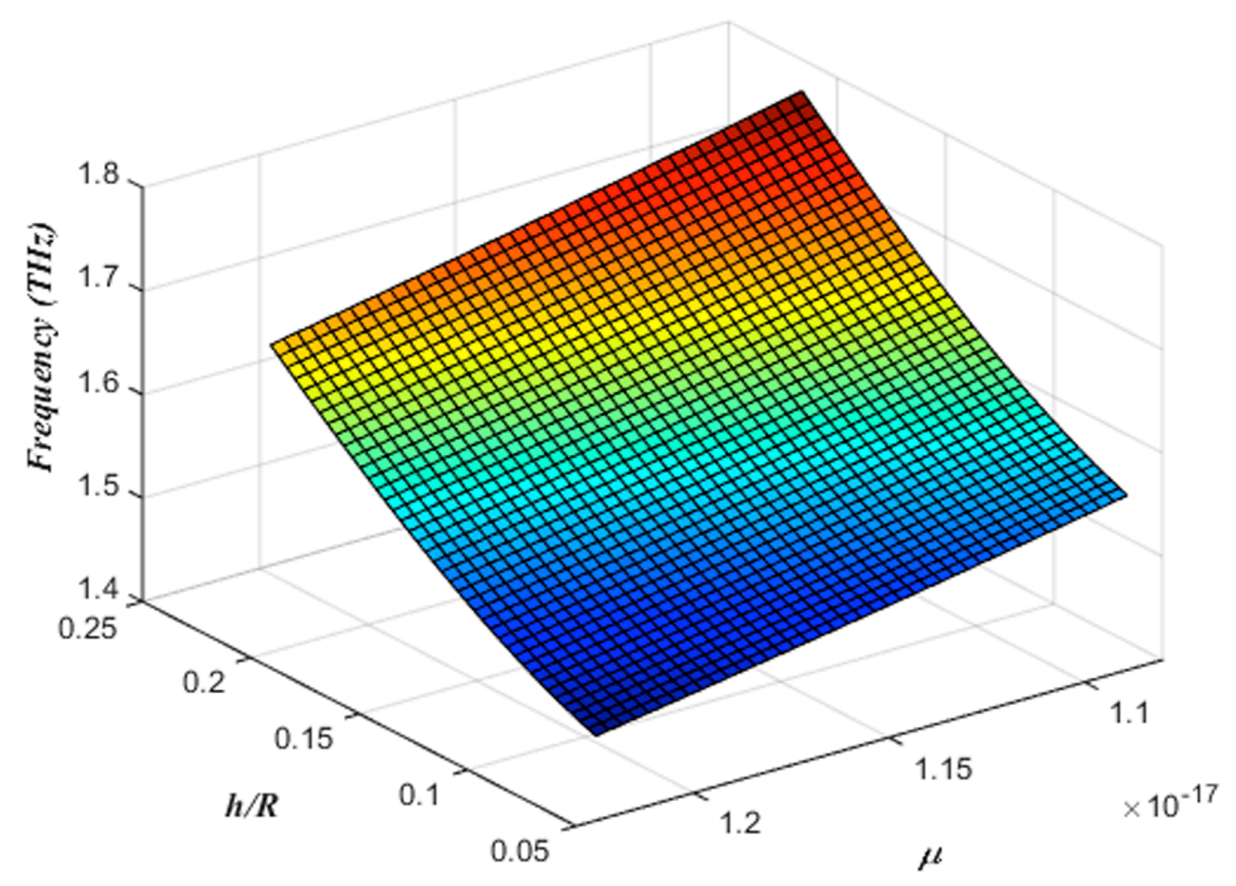

Fig. 6. Effect of thickness ratio on the frequency for different nonlocal parameters $(\beta=2, \eta=$ $\left.(0.4 \mathrm{e}-9)^{2}\right)$.

Variation of frequency versus length ratio for different power law index is illustrated in Fig. 7. As is evident from Fig. 7, the frequency is shown to be decreasing with increasing length ratio and this effect is more significant by increasing power law index which depicting stiffer nanotubes. In other words, the effects of length ratio on the frequency with greater power law index are relatively more than those of ones with small power law index.

In order to have a deeper insight into the influence of length ratio, Figs. 8 and 9 are also illustrated for various scale factors and nonlocal parameters. According to these figures, the decreasing procedure of frequency with respect to the increase in length ratio for various scale factors and nonlocal parameters is the same as Fig. 7. Moreover, from these figures it can be seen that the influence of scale factor and nonlocal parameter is more evident when length ratio is small. Also, according to Figs. 8 and 9, at high length ratio the results of the present model approach to those of classical ones which shows the capability of classical model to predict the vibration response of large-scale structures. 


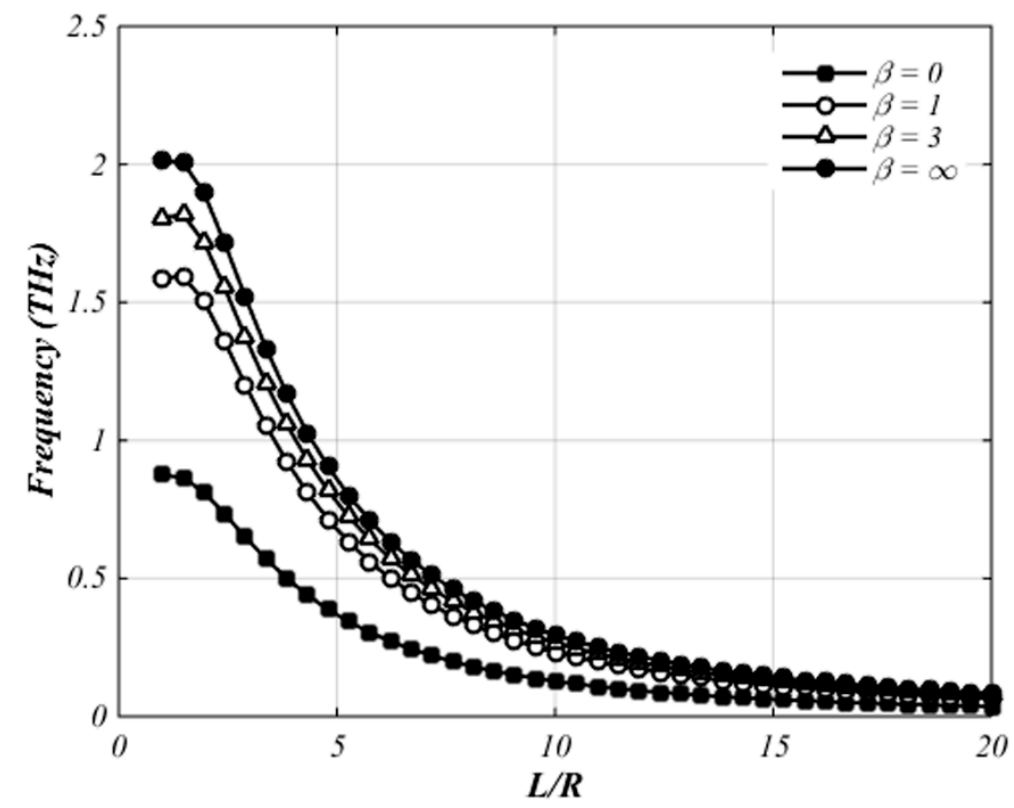

Fig. 7. Effect of length ratio on frequency for different power law index $\left(\mu=(3.3 e-9)^{2}, \eta=(0.4 e-9)^{2}\right)$.

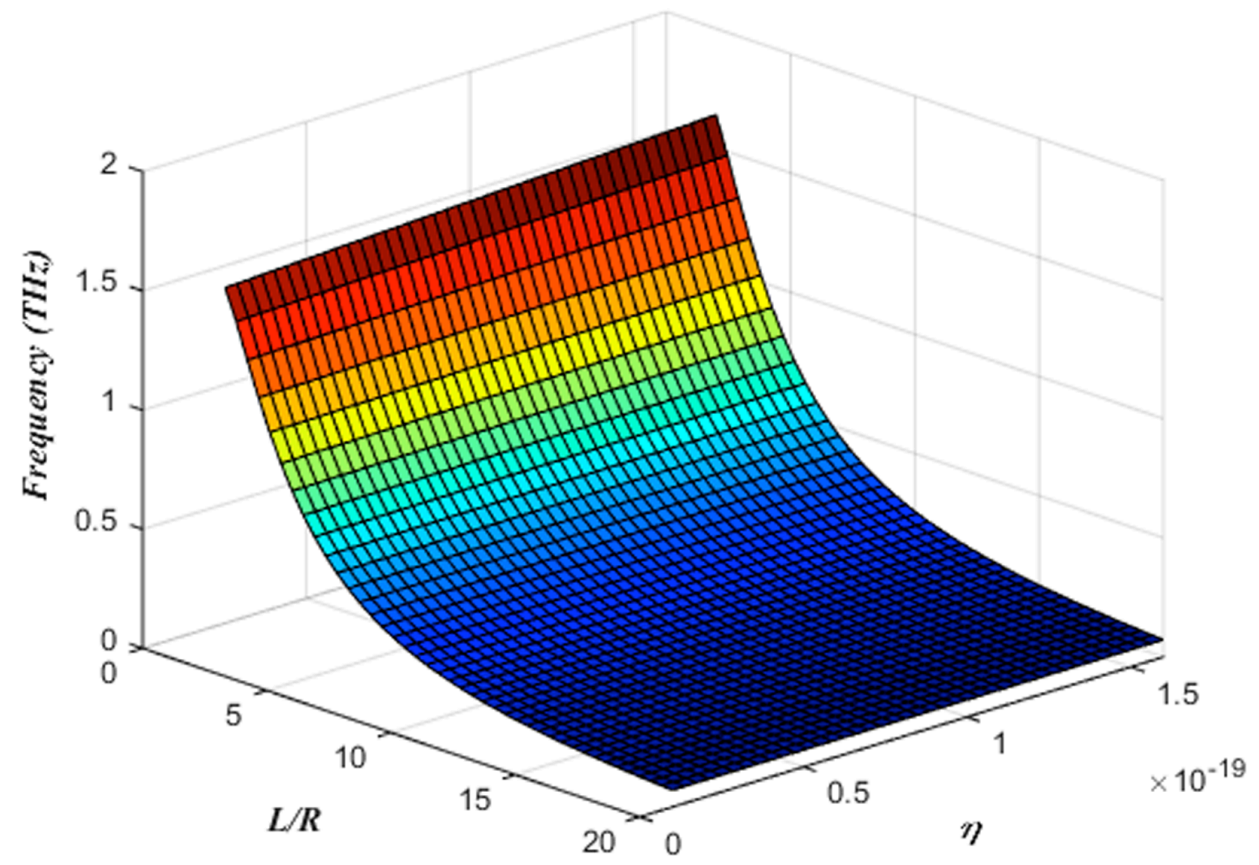

Fig. 8. Effect of length ratio on frequency for different scale factors $\left(\beta=2, \mu=(3.3 e-9)^{2}\right)$. 


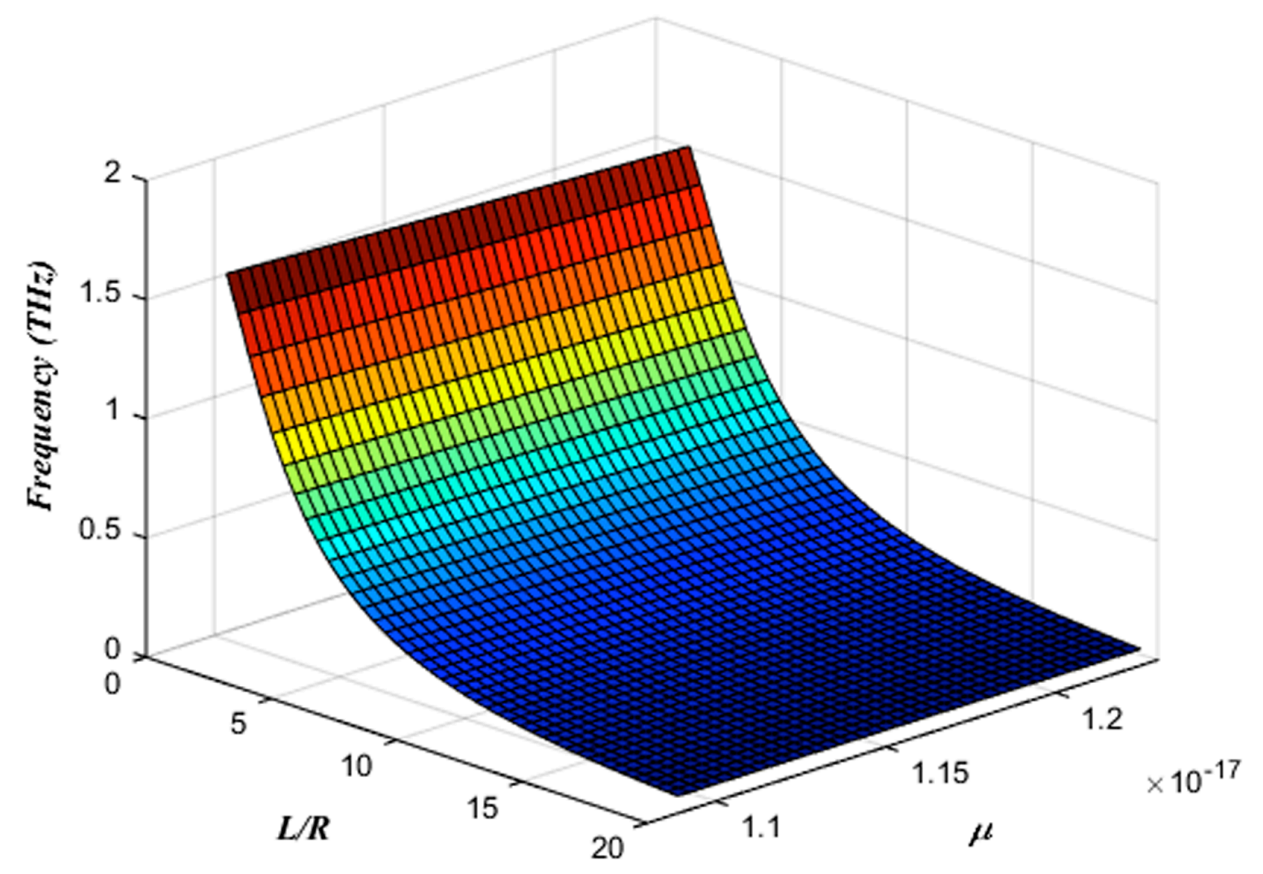

Fig. 9. Effect of length ratio on frequency for different nonlocal parameters $\left(\beta=2, \eta=(0.4 \mathrm{e}-9)^{2}\right)$.

\section{Conclusion}

In this study, the free vibration of FG nanotube is studied based on the nonlocal strain gradient theory and first order shear deformable theory. The material properties are considered to be variable through thickness direction according to power law distribution. The governing equations and boundary conditions are derived based on the Hamilton's principle and the free vibration of simply supported FG nanotube is studied as well. The effects of various parameters such as material length scale parameters, thickness, length and power law index are investigated on the frequency. It was revealed that increase in power law index intensifies the influence of nonlocal parameter and scale factors on the FG nanotube frequency. Moreover, the higher frequency appears at higher thickness ratios and lower length ratios. Furthermore, the effects of length ratio and thickness ratio are relatively intense for greater scale factors and lower nonlocal parameters.

\section{References}

[1] Tadi Beni, Y., Mehralian, F., Zeighampour, H., The modified couple stress functionally graded cylindrical thin shell formulation. Mechanics of Advanced Materials and Structures, 23(7), 791-801, 2016.

[2] Tadi Beni, Y., Mehralian, F., Razavi, H., Free vibration analysis of size-dependent shear deformable functionally graded cylindrical shell on the basis of modified couple stress theory. Composite Structures, 120, 65-78, 2015. 
[3] Li, X., Li, L., Hu, Y., Ding, Z., Deng, W., Bending, buckling and vibration of axially functionally graded beams based on nonlocal strain gradient theory. Composite Structures, 165, 250-265, 2017.

[4] Ebrahimi, F., Barati, M.R., Dabbagh, A., A nonlocal strain gradient theory for wave propagation analysis in temperature-dependent inhomogeneous nanoplates. International Journal of Engineering Science, 107, 169-182, 2016.

[5] Civalek, Ö., Buckling analysis of composite panels and shells with different material properties by discrete singular convolution (DSC) method. Composite Structures, 161, 93$110,2017$.

[6] Akgöz, B., and Civalek, Ö., Longitudinal vibration analysis of strain gradient bars made of functionally graded materials (FGM). Composites Part B: Engineering, 55, 263-268, 2013.

[7] Dzenis, Y., Spinning continuous fibers for nanotechnology. Science, 304 (5679), 19171919, 2004.

[8] Love, J.C., Estroff, L.A., Kriebel, J.K., Nuzzo, R.G., Whitesides, G.M., Self-assembled monolayers of thiolates on metals as a form of nanotechnology. Chemical reviews, 105(4), 1103-1170, 2005.

[9] Mehralian, F., Tadi Beni, Y., Ansari, R. Size dependent buckling analysis of functionally graded piezoelectric cylindrical nanoshell. Composite Structures, 152, 45-61, 2016.

[10] Gholami, R., Darvizeh, A., Ansari, R., Hosseinzadeh, M., Size-dependent axial buckling analysis of functionally graded circular cylindrical microshells based on the modified strain gradient elasticity theory. Meccanica 49(7), 1679-1695, 2014.

[11] Mehralian, F., Tadi Beni, Y., Thermo-Mechanical vibration of size dependent shear deformable functionally graded conical nanoshell resting on elastic foundation. International Journal of Engineering \& Applied Sciences, 8(2), 68-86, 2016.

[12] Mercan, K., Civalek, Ö., Buckling analysis of Silicon carbide nanotubes (SiCNTs) with surface effect and nonlocal elasticity using the method of HDQ. Composites Part B: Engineering, 114, 34-45, 2017.

[13] Akgöz, B., Civalek, Ö., Buckling analysis of cantilever carbon nanotubes using the strain gradient elasticity and modified couple stress theories. Journal of Computational and Theoretical Nanoscience, 8(9), 1821-1827, 2011.

[14] Lim, C.W., Zhang, G., Reddy, J.N., A higher-order nonlocal elasticity and strain gradient theory and its applications in wave propagation. Journal of the Mechanics and Physics of Solids, 78, 298-313, 2015.

[15] Ebrahimi, F., Barati, M.R., A nonlocal strain gradient refined beam model for buckling analysis of size-dependent shear-deformable curved FG nanobeams. Composite Structures, 159, 174-182, 2017.

[16] Tang, Y., Liu, Y., Zhao, D., Wave dispersion in viscoelastic single walled carbon nanotubes based on the nonlocal strain gradient Timoshenko beam model. Physica E: Lowdimensional Systems and Nanostructures, 87, 301-307, 2017. 
[17] Sahmani, S., Ansari, R., Gholami, R., Darvizeh, A., Dynamic stability analysis of functionally graded higher-order shear deformable microshells based on the modified couple stress elasticity theory. Composite Part B, 51, 44-53, 2013.

\section{Appendix A}

$$
\begin{aligned}
& \delta U=0 \quad \text { or } \quad N_{x x}-\frac{1}{R} \frac{\partial N_{x \theta}^{(1)}}{\partial \theta}=0 \\
& \frac{\partial \delta U}{\partial x}=0 \quad \text { or } \quad N_{x x}^{(1)}=0 \\
& \delta V=0 \quad \text { or } \quad N_{x \theta}-\frac{1}{R} \frac{\partial N_{\theta \theta}^{(1)}}{\partial \theta}-\frac{Q_{\theta z}^{(1)}}{R}=0 \\
& \frac{\partial \delta V}{\partial x}=0 \quad \text { or } \quad N_{x \theta}^{(1)}=0 \\
& \delta W=0 \quad \text { or } \quad Q_{x z}+\frac{N_{\theta \theta}^{(1)}}{R}-\frac{1}{R} \frac{\partial Q_{\theta z}^{(1)}}{\partial \theta}=0 \\
& \frac{\partial \delta W}{\partial x}=0 \quad \text { or } \quad Q_{x z}^{(1)}=0 \\
& \delta \psi_{x}=0 \quad \text { or } \quad M_{x x}-\frac{1}{R} \frac{\partial M_{x \theta}^{(1)}}{\partial \theta}+Q_{x z}^{(1)}=0 \\
& \frac{\partial \delta \psi_{x}}{\partial x}=0 \quad \text { or } \quad M_{x x}^{(1)}=0 \\
& \delta \psi_{\theta}=0 \quad \text { or } \quad M_{x \theta}-\frac{1}{R} \frac{\partial M_{\theta \theta}^{(1)}}{\partial \theta}+Q_{\theta z}^{(1)}=0 \\
& \frac{\partial \delta \psi_{\theta}}{\partial x}=0 \quad \text { or } \quad M_{x \theta}^{(1)}=0
\end{aligned}
$$

Due to the stress distribution along thickness of the shell, stress resultants are introduced as follows:

$$
\begin{aligned}
& {\left[\begin{array}{c}
N_{x x}, N^{(1)}{ }_{x x} \\
N_{x \theta}, N^{(1)}{ }_{x \theta} \\
N_{\theta \theta}, N^{(1)}{ }_{\theta \theta}
\end{array}\right]=\int_{-\hat{z}_{c}}^{h-\hat{z}_{c}}\left\{\begin{array}{l}
t_{x x}, \sigma^{(1)}{ }_{x x}, \\
t_{x \theta}, \sigma^{(1)}{ }_{x \theta} \\
t_{\theta \theta}, \sigma^{(1)}{ }_{\theta \theta}
\end{array}\right\} d z} \\
& {\left[\begin{array}{l}
Q_{z x}, Q^{(1)}{ }_{z x} \\
Q_{z \theta}, Q^{(1)}{ }_{z \theta}
\end{array}\right]=\int_{-\hat{z}_{c}}^{h-\hat{z}_{c}}\left\{\begin{array}{l}
t_{z x}, \sigma^{(1)}{ }_{z x} \\
t_{z \theta}, \sigma^{(1)}{ }_{z \theta}
\end{array}\right\} d z} \\
& {\left[\begin{array}{l}
M_{x x}, M^{(1)}{ }_{x x} \\
M_{\theta \theta}, M^{(1)}{ }_{\theta \theta}
\end{array}\right]=\int_{-\hat{z}_{c}}^{h-\hat{z}_{c}}\left\{\begin{array}{l}
t_{x x}, \sigma^{(1)}{ }_{x x}, \sigma_{\theta \theta}^{(1)} \\
t_{\theta \theta}
\end{array}\right\} z d z}
\end{aligned}
$$

